\title{
BMJ Open Assessing the effectiveness and implementation of a chronic disease self-management programme in faith- based organisations in Barbados: protocol for a cluster randomised parallel trial
}

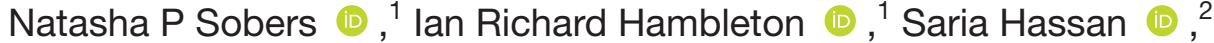 \\ Simon George Anderson (D) , ${ }^{1}$ Lisa Brathwaite-Graham, ${ }^{1}$ Kia Lewis, ${ }^{1}$ \\ Trevor S Ferguson ${ }^{3}{ }^{3}$
}

To cite: Sobers NP, Hambleton IR, Hassan S, et al. Assessing the effectiveness and implementation of a chronic disease selfmanagement programme in faith-based organisations in Barbados: protocol for a cluster randomised parallel trial. BMJ Open 2021;11:e050548. doi:10.1136/ bmjopen-2021-050548

- Prepublication history and additional supplemental material for this paper are available online. To view these files, please visit the journal online (http://dx.doi.org/10.1136/ bmjopen-2021-050548)

Received 24 February 2021 Accepted 10 September 2021

D) Check for updates

(c) Author(s) (or their employer(s)) 2021. Re-use permitted under CC BY-NC. No commercial re-use. See rights and permissions. Published by BMJ.

For numbered affiliations see end of article.

\section{Correspondence to}

Dr Natasha P Sobers;

natasha.sobers@cavehill.uwi. edu

\section{ABSTRACT}

Introduction In the COVID-19 environment of reduced patient interaction with the healthcare system, evidencedbased self-care of chronic disease is vital. We will evaluate the effect of an online chronic disease self-management programme (CDSMP) plus medication adherence tools on systolic blood pressure (SBP) (primary aim) and, seek to understand the barriers and facilitators to implementation of this modified CDSMP in faith-based organisations (FBOs) (secondary aim).

Methods We will conduct an unblinded cluster randomised trial in FBOs throughout Barbados. Eligibility: Persons ages 35-70 years; a previous diagnosis of hypertension or currently on antihypertensive therapy and the occurrence of two or more blood pressure readings above $130 \mathrm{~mm} \mathrm{Hg}$ (systolic) or $80 \mathrm{~mm} \mathrm{Hg}$ (diastolic) on the day of recruitment. Persons not known to have hypertension but who have two or more blood pressure readings at or above $130 \mathrm{~mm} \mathrm{Hg}$ (systolic) or $80 \mathrm{~mm} \mathrm{Hg}$ (diastolic) on two recruitment days at least 1 week apart will also be eligible. The unit of randomisation is a church cluster which consists of 7-9 churches. We will perform block randomisation to assign 24 clusters to intervention or control. The intervention has three components: modified CDSMP workshops, distribution of medication pill boxes and use of social media (WhatsApp V.2.0) to encourage medication adherence. Controls will receive one didactic lecture only. We will determine the mean changes in SBP levels for the intervention group versus controls and compare differences in outcomes 6 months' post intervention using mixed effects regression models. Ethics and dissemination This project has received ethical approval from the Institutional Review Board of the University of the West Indies in Barbados. Dissemination will use peer-reviewed publications, policy briefs to government and guidelines to leaders of FBOs. We aim to increase the proportion of patients with controlled hypertension and inform implementation of selfmanagement programmes in small populations. Trail registration number NCT04437966
Strengths and limitations of this study

- This is the first evaluation of the virtual delivery of a Chronic Disease Self-management Programme with medication adherence tools in the Caribbean.

- The study will leverage the social support mechanisms existing within faith-based organisations (FBOs) to disseminate an evidence-based selfmanagement intervention for hypertension.

- The inclusion of improved medication adherence strategies is likely to improve hypertension control rates by at least $10 \%$.

- Our findings on factors impacting implementation should be transferable to other small island developing states/territories with close-knit vibrant FBOs.

- Engagement of FBOs is likely to under-represent men, as FBO membership in our trial sites consist of more than $70 \%$ women.

\section{BACKGROUND AND SIGNIFICANCE}

Hypertension is the single most important contributor to morbidity and mortality associated with cardiovascular diseases. ${ }^{1}$ A systematic review of hypertension prevalence in low-income and middle-income countries (LMICs), reported prevalence rates for the Latin America and the Caribbean region that were the highest of the regions studied-39.1\%, compared with $32 \%$ average among LMICs. ${ }^{2}$ In Barbados, prevalence levels of hypertension $(40.7 \%)$ are similarly high and account for the highest proportion of deaths due to cardiovascular diseases. ${ }^{34}$ The financial burden is high: combined, cardiovascular disease (hypertension, heart attack and stroke) and diabetes were estimated to cost the Barbadian economy approximately US\$105 million per year $(\sim 3.5 \%$ 
of Gross Domestic Product (GDP)). ${ }^{5}$ Despite the high morbidity and financial burden, treatment and control rates are suboptimal. In Barbados, approximately one in three women and one in five men with hypertension were treated. Of those who were being treated, roughly $40 \%$ were found to have sub-optimal blood pressure, implying that they would benefit from further control measureswhether increased medication, changes in lifestyle or both. ${ }^{3}$

Recent systematic reviews provide consistent evidence that hypertension self-management reduces blood pressure. ${ }^{7-9}$ Despite this, evidenced-based patient selfmanagement programmes are uncommon in most LMICs and small island developing states (SIDS) with an acute need for low cost initiatives. In the COVID-19 environment associated with reductions in patient interaction with the healthcare system in many countries, evidencedbased structured self-care takes on a new importance. The focus of Chronic Disease Self-management programmes (CDSMPs) is to promote skills that facilitate the patient's management of their own disease and its associated challenges. The CDSMP managed by the Self-Management Resource Centre (SMRC) has been shown to be effective in improving self-management and health service utilisation. ${ }^{1011}$

Given the strong evidence for the ability of this CDSMP to improve chronic disease care, we propose to translate this programme to the Caribbean, leveraging faith-based organisations (FBOs) to deliver the programme. Attempts have been made to implement CDSMP programmes in the Eastern Caribbean, but the programmes are not yet sustained and are largely clinic-led rather than community based. The lack of consistent delivery of the programme in this region provides an opportunity to understand the facilitators and barriers to communitybased implementation.

We aim to deliver a multipronged hypertension selfmanagement programme in the community-based setting of FBOs. FBOs are being used increasingly for health interventions, particularly in African descent populations. ${ }^{12}{ }^{13}$ With over 300 churches in 166 square miles, and $80 \%$ of population having a religious affiliation, ${ }^{14} \mathrm{FBOs}$ in Barbados provide a structured way in which to engage the community. Furthermore, public health and FBOs tend to have common goals of promoting equity and justice. Our objectives are as follows. We will adapt the established CDSMP making culturally appropriate modifications. To enhance the effect of the programme on blood pressure control we will add and evaluate the following medication adherence strategies: pill boxes, electronic medication adherence reminders and education on home blood pressure monitoring. ${ }^{15}{ }^{16}$ We will assess the effectiveness of the adapted CDSMP programme plus the medication adherence tools on systolic blood pressure (SBP) (primary aim) and seek to understand the factors influencing implementation of the programme in FBOs (secondary aim). This will help to inform potential scale up conditional on the effectiveness of the intervention in this setting.
METHODS

\section{Setting}

This cluster randomised trial will be conducted among members of selected church groups in Barbados-a SIDS in the Caribbean with a population of $287025(52 \%$ female). According to the Barbados 2010 census, approximately three-quarters of the population identify as Christian. ${ }^{17}$ This study is intended for Christian churches in Barbados, from any denomination. Barbados is divided into 11 parishes. Given the small sizes of many churches in Barbados we have chosen to cluster by parish. Ten of the parishes will be divided into two, while the most densely populated parish (St. Michael) will be divided into four divisions. This gives us a total of 24 geographical divisions. Each division consists of 7-9 of our target churches comprising a total of about 350 members per division. The study will consider each division as a single unit which will be randomised to either intervention or control. The treatment of a division as one assembly allows the study to have sufficient participants at each recruitment event to be able to meet the required sample size of persons with uncontrolled hypertension.

\section{Eligibility criteria}

All persons 35-70 years who are attendees of the FBOs will be eligible to participate. Eligible persons must have been resident in Barbados for the previous 6 months and not planning to migrate over the ensuing 1 year (figure 1 ). Potential participants will be invited to recruitment days where they are screened for hypertension. All persons with a previous diagnosis of hypertension or currently on antihypertensives and the occurrence of two or more blood pressure readings at or above $130 \mathrm{~mm} \mathrm{Hg}$ (systolic) or $80 \mathrm{~mm} \mathrm{Hg}$ (diastolic) on the day of recruitment will be deemed eligible. Persons not known to have hypertension but who have two or more blood pressure readings

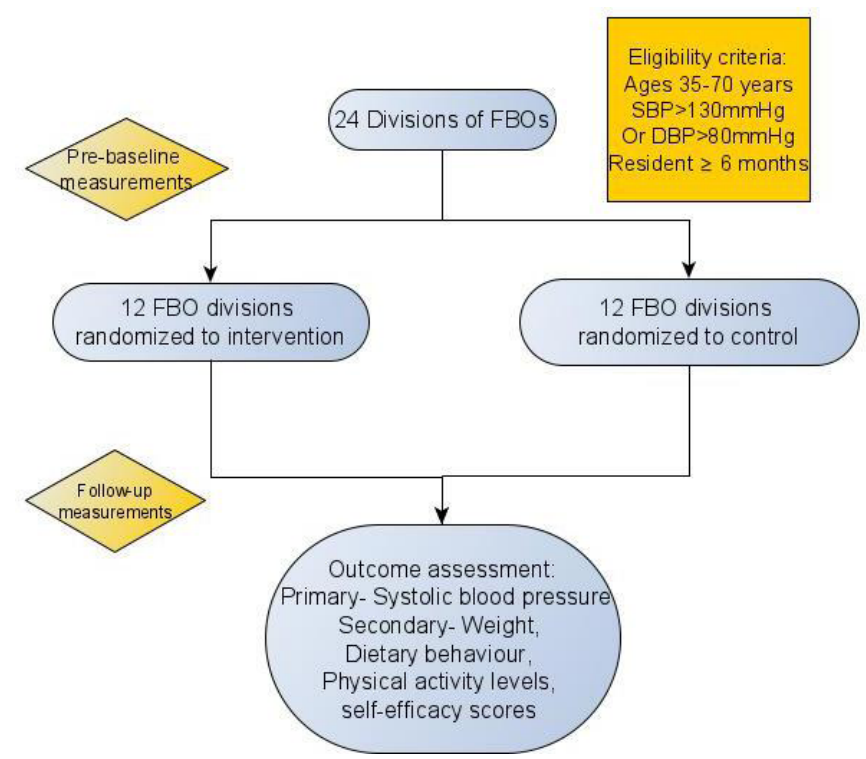

Figure 1 Patient flow and selection. FBO, faith-based organisation; DBP, diastolic blood pressure; SBP, systolic blood pressure. 
at or above $130 \mathrm{~mm} \mathrm{Hg}$ (systolic) or $80 \mathrm{~mm} \mathrm{Hg}$ (diastolic) on the day of recruitment are also eligible. These goals and diagnostic criteria for SBP and diastolic blood pressure control are in keeping with American Heart Association/American College of Cardiology 2017 Hypertension Guidelines. ${ }^{18}$

\section{Exclusion criteria}

Persons who are well controlled on blood pressure lowering drugs will be excluded. Other exclusion criteria are as follows: known participation in another study, unwillingness/inability to comply with study protocol and pregnancy or plans to become pregnant within the next 9 months.

Screening will be conducted by volunteer healthcare providers (doctors, nurses, nursing auxiliaries) who are members of the churches participating in the study. Churches which do not have a doctor or nurse as part of the membership will be assigned a healthcare provider from within the same division. Screening and data collection teams at each church will receive training on appropriate blood pressure measurement techniques in alignment with recommendations in the 2020 Global International Society of Hypertension guidelines. Data collectors will be required to complete a course delivered by the Pan American Health Organization-Virtual Course on accurate automated blood pressure measurement (2020). ${ }^{19}$ Teams at each church will be given Omron devices and appropriate cuff sizes. The full protocol for blood pressure measurement is available in online supplemental appendix 1. Measurement of weight and height will be conducted using SECA scales and stadiometers, respectively.

\section{Recruitment}

To obtain eligible participants, flyers and information on the study will be circulated on social media and in regular church announcements and notice boards. Organisations will host one or more recruitment days at local churches inviting all members of the community to participate. Recruitment days will be conducted with strict physical distancing protocols in place which are in keeping with the stage of restriction Barbados is experiencing at the time of recruitment. Recruitment days will be held before and after regular church services and in accordance with COVID-19 directives for churches, issued by the Ministry of Health and Wellness. Prospectively, recruitment will not occur during times of curfew/lockdown and will conform to government directives. As of December 2020, churches in Barbados were allowed to hold services given that attendees are at least $1 \mathrm{~m}$ apart, wearing face masks throughout with temperatures taken and hand sanitising required before entry to the building. Where possible, volunteer health personnel are encouraged at all services. ${ }^{20}$ Recruitment was paused in January 2021 when public health directives prevented gatherings of more than 10 persons. This will recommence when COVID-19 directives allow church gatherings. Members and community affiliates will be invited to have their blood pressure measurements and weights at their church and persons fitting the eligibility criteria will be invited to take part in the study. Once eligibility is determined, potential participants will be consented on the day of recruitment when anthropometric measurements are taken (online supplemental appendix 2). Consented participants will be called (via telephone) at a later date to participate in an initial $30 \mathrm{~min}$ interview by the study data collectors.

\section{Randomisation and blinding}

Churches will be assigned to one of 24 clusters based on geographical location and will be randomised using block randomisation with block sizes of four. The study statistician will assign each church division to either intervention or control groups based on a 1:1 ratio, using a prespecified random number generator (Stata statistical software V.16). All churches ${ }^{7-9}$ within a division will receive the intervention or control according to their random allocation. Given the nature of the intervention, the trial will be unblinded. However, we will implement allocation concealment among study data collectors, who will have no a priori knowledge of church randomisation status until the end of their recruitment. The study is a parallel design.

\section{Sample size}

Previous studies have found group mean SBP reductions ranging from $4.5 \mathrm{~mm} \mathrm{Hg}$ to $6.6 \mathrm{~mm} \mathrm{Hg} .{ }^{1321}{ }^{22}$ Using the lowest group mean SBP reduction of $4.5 \mathrm{~mm} \mathrm{Hg}$ (SD $10)^{22}$ among the persons living with uncontrolled hypertension and given a study of 24 clusters we estimated that 132 persons in the intervention and 132 in the control group, would provide $80 \%$ power to detect a difference of $4.5 \mathrm{~mm} \mathrm{Hg}$. This is assuming an intracluster correlation (ICC) of $0.04 .^{13}$ This required sample size of 264 provides us with cluster sizes of 11 . ICCs in various school and primary care clinic studies have been shown to be small and positive. ${ }^{23}$ Calculations were performed using Stata statistical software V.16. ${ }^{24}$

\section{Description of intervention}

The intervention consists of three components: (1) CDSMP, (2) distribution of and teaching on the use of medication pill boxes and (3) use of social media (WhatsApp V.2.0) to encourage medication adherence (table 1). We combine previously tested CDSMP and add medication adherence tools and social media use to develop a novel intervention focusing on improving patient self-efficacy to manage and make decisions about their illness. The SMRC managed CDSMP minimises traditional methods of didactic knowledge transfer and focuses on enhancing skills through problem solving, decision making, goal setting and brainstorming activities. ${ }^{25}$ The programme consists of a workshop which runs for 6 weeks for 2.5 hours per week. Since the onset of COVID-19 the master trainers in the Caribbean have been recertified to deliver the course virtually. Each 
Table 1 Description of intervention components

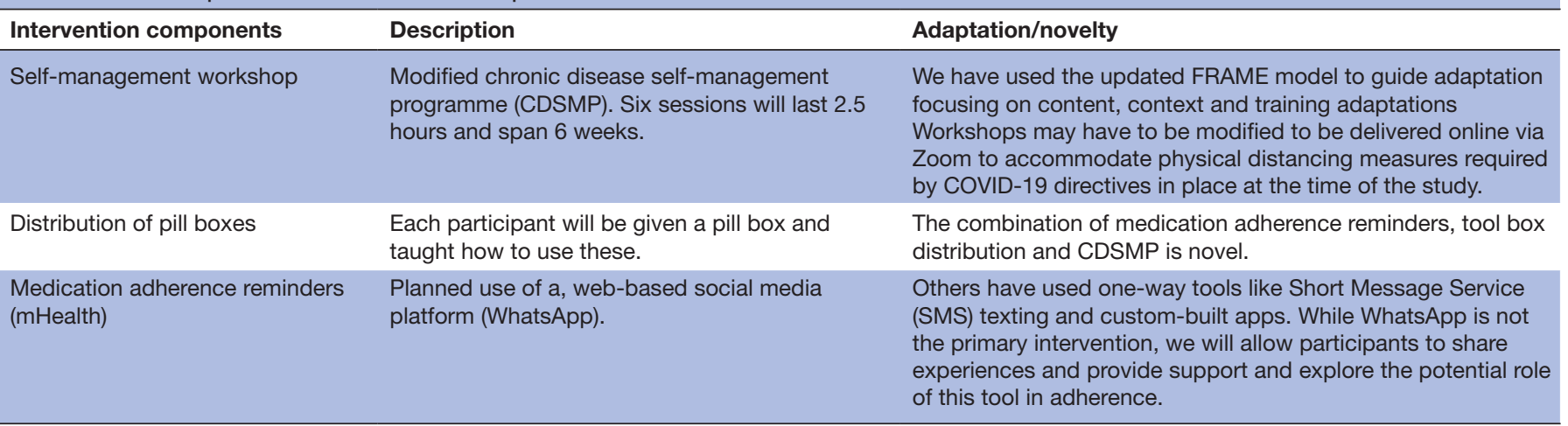

participant will be guided with the use of the book entitled 'Living a Healthy Life with Chronic Conditions, ${ }^{26}$ and taken through self-management tools presented in a chart called the Self-Management Toolbox. These tools focus on: physical activity, medications, decision making, action planning, breathing techniques, understanding emotions, problem solving, using your mind, sleep, communication, healthy eating, weight management, working with health professionals.

To adapt the CDSMP to ensure cultural appropriateness to Barbados we held a series of stakeholder engagement meetings with CDSMP master trainers, previous participants of a diabetes self-management workshop designed for Barbados, church leaders and a Barbadian nutritionist (table 2). The Wiltsey Stirman et al model was used to guide the adaptation process, allowing us to systematically look at content, context and training adaptations. ${ }^{27}$ In the stakeholder meetings, we discussed the original lifestyle workshop series, and what adaptations were needed to make it more appropriate and relevant to the Caribbean.

To maintain fidelity to the CDSMP programme, all components of the original programme which includes blood pressure control, finding and affording healthy foods, label reading, physical activity, planning a healthy plate, making traditional foods healthy and portion control will be delivered. An additional session will be added at the beginning of the workshop series, which will place emphasis on knowledge of hypertension and antihypertensives. Tips for enhancing medication adherence will be emphasised as well as ways to handle common side effects of drugs used to treat hypertension. This session will be referred to as session zero. Minor nutrition modifications will be made to the main sessions by using foods

Table 2 Characteristics of planned preimplementation adaptations targeted at the intervention group

\begin{tabular}{llll}
\hline Description and goal of adaptation & $\begin{array}{l}\text { Who participated in the decision } \\
\text { to modify? }\end{array}$ & $\begin{array}{l}\text { What is modified? (Nature of } \\
\text { content modification) }\end{array}$ & $\begin{array}{l}\text { Relationship fidelity/core } \\
\text { elements }\end{array}$ \\
\hline $\begin{array}{l}\text { Adjustments to dietary examples } \\
\text { GOAL: Enhanced cultural fit }\end{array}$ & $\begin{array}{l}\text { Nutritionist } \\
\text { Study Researchers }\end{array}$ & $\begin{array}{l}\text { CONTENT: Change in dietary } \\
\text { examples using foods familiar to } \\
\text { Barbadians (eg, yams, breadfruit) } \\
\text { (Tailoring) }\end{array}$ & $\begin{array}{l}\text { Fidelity consistent/core elements } \\
\text { or functions preserved }\end{array}$ \\
& & CONTEXT: Online delivery &
\end{tabular}

Additional information on hypertension and medication adherence GOAL: Enhanced intervention effectiveness(Improved blood pressure control)

\section{CDSMP Master trainers}

Former self-management workshop participant FBO leaders

Implementation consultant Study researchers

Programme manager

\section{CONTENT: Additional session at}

start of workshop series to introduce hypertension, its complications, importance of medication adherence and dealing with side effects (Adding elements) CONTEXT: Online delivery TRAINING: CDSMP workshop leaders will need two additional hours of training to become familiar with the content of session 1.

$\begin{array}{ll}\text { Complete delivery of CDSMP } & \text { Study researchers } \\ \text { programme via Zoom } & \text { FBO leaders } \\ \text { GOAL: Facilitate safe delivery during the } & \text { CDSMP master trainers } \\ \text { SARS-COV2 viral pandemic } & \text { Self-management Resource Centre } \\ & \text { (SMRC) }^{*}\end{array}$

\section{CONTENT: No changes made to} standard CDSMP programme as outlined by SMRC.

CONTEXT: Online delivery TRAINING: Workshop training now done online with a change in structure to facilitate this format. For example, twice weekly training sessions rather than once.
Fidelity consistent: core CDSMP methodology preserved despite additional elements.

Fidelity consistent: core elements of the programme have been retained. 
and packaging commonly used in Barbados as well as the Caribbean Food and Nutrition Pyramids will be highlighted in the nutrition aspect of the programme. In view of the need to adhere to physical distance guidelines in the era of COVID-19 disease, the entire programme will be delivered virtually. This study represents the first studied virtual delivery of the programme in the Caribbean.

The church leaders provided significant insight into addressing challenges that may arise with participants' attendance at online sessions indicating that sessions can be held before workshops to familiarise participants with the virtual platform and suggested the use of technical assistants to support workshop content leaders. Each workshop will consist of exercises where participants are required to: plan changes for the upcoming week (action planning); provide feedback on the prior week and attempt to solve any challenges faced during that week (problem solving); brainstorm solutions to actual or potential problems. Church volunteers with chronic diseases will be trained as workshop leaders. The training programme is designed for persons with no previous healthcare training and has been found to be most effective when the leaders have a chronic disease.

Medication adherence: Pill boxes will be distributed to all individuals in the intervention group. Two medical doctors and one nurse attached to the study as principal investigator and research assistants will teach participants to use the pillboxes. During the workshop series, participants will be sent twice weekly reminders to use their high blood pressure medications via the social media tool WhatsApp. Our intention is to offer a networking solution that minimises the number of participants needing to adopt a new social networking technology-and this was a driving force for choosing WhatsApp software. As of August 2021, WhatsApp continues to operate end-to-end encryption of all message content, and so meets our encryption standards for RCT operation. A World Bank report indicates high cell phone penetration in Barbados (115 cell phones per 100 persons) as well as one of the highest internet speeds in Caribbean. ${ }^{28}$ Participants will also be allowed to share messages regarding experiences with adherence and provide peer support. Controls will receive one didactic session (on importance of medication adherence to hypertension treatment) lasting 1 hour delivered by a healthcare professional. Controls will be derived from geographic divisions not randomised to the intervention and will receive questionnaires at intervals identical to the intervention group.

\section{Data collection tools}

During an interview using virtual media with both video and audio capabilities, data will be collected in the domains noted in table 3 . We will use a modified version of the freely available Dietary Screening Questionnaire (DSQ) suitable for use via telephone. ${ }^{29}$ This DSQ has also been used in population-based cross-sectional studies being conducted in Barbados and would facilitate comparisons. Using the DSQ, we will calculate the Dietary Approaches to Stop Hypertension (DASH) score based on methods used by Fung et al. ${ }^{30}$ Global Physical Activity questionnaire will be used to assess physical activity; the Self-Efficacy for Managing Chronic Diseases six-item Scale developed by Lorig et al to assess self-efficacy ${ }^{31}$ and the Hill-Bone Compliance to High Blood pressure therapy scale ${ }^{32}$ for medication adherence. The latter is validated in a Black population and developed specifically for hypertension. This tool will be compared with pill counts before and after the intervention. Immediately after the final workshop of the intervention, blood pressure, weight, physical activity survey and dietary screening assessments will be repeated. Each control site will receive repeat measurements after the workshops are complete. At each intervention site and control site, patients will be contacted 6 months after the first day of their workshop for repeat of weight, blood pressure, dietary habits, physical activity, medication adherence and self-efficacy. Surveys will be conducted virtually to minimise the need for in person contact and reduce the risk of transmission COVID-19.

\section{Describing evaluation of implementation}

Given the proven effectiveness of the CDSMP programme in other countries, we will also study factors related to implementation in this context. To facilitate this evaluation of implementation we will use the RE-AIM framework which uses five dimensions to assess impact: reach, effectiveness, adoption, implementation and maintenance. ${ }^{33}$ RE-AIM has been chosen because it addresses issues at both the individual and organisational/setting level and because of its high construct flexibility. We will assess reach as the proportion of eligible participants who participate in the program and will also assess representativeness by comparing participant characteristics with a Barbadian population-based cross-sectional survey. Effectiveness will be assessed using the change in mean SBP between intervention and control groups as described above. Adoption will be evaluated as the proportion of churches randomised to the intervention who were able to implement the programme. We will assess the impact of size of church, denomination and presence of a previous health committee or programme on adoption. We will compare the characteristics of those engaged versus those not engaged to understand potential barriers to reach. We will use number of workshops attended and number of messages sent on WhatsApp as markers of engagement and assess the impact of engagement on effectiveness after adjustment for socio-demographic characteristics (eg, age sex, marital status).

Adoption will also be assessed at the individual level looking at the proportion of individuals who are trained to lead the workshops who ultimately engage in the programme. We will also assess the number of churches which had persons willing to volunteer to be trained to monitor and conduct blood pressure screening and become workshop leaders. We will assess implementation in the intervention churches by evaluating the fidelity to the intervention components. Our monitoring of fidelity 
Table 3 Timeline for measurements

\begin{tabular}{|c|c|c|c|c|}
\hline Study measures & Pre-randomisation visit & Pre-workshop survey & Post-workshop & Six months \\
\hline \multicolumn{5}{|l|}{ Anthropometric measures } \\
\hline $\begin{array}{l}\text { Blood pressure } \\
\text { measurements }\end{array}$ & $\mathbf{x}$ & & $\mathbf{x}$ & $\mathbf{x}$ \\
\hline Height and weight & $x$ & & $x$ & $x$ \\
\hline \multicolumn{5}{|l|}{ Questionnaire measures } \\
\hline Demographics & $x$ & & & \\
\hline Alcohol use & & $x$ & $x$ & $x$ \\
\hline Fruit and vegetable intake & & $x$ & $\mathbf{x}$ & $x$ \\
\hline $\begin{array}{l}\text { Dietary intake (Dietary } \\
\text { Screening Questionnaire) }\end{array}$ & & $x$ & $\mathbf{x}$ & $\mathbf{x}$ \\
\hline $\begin{array}{l}\text { Physical activity (Global } \\
\text { Physical Activity } \\
\text { Questionnaire) }\end{array}$ & & $x$ & & $\mathbf{x}$ \\
\hline $\begin{array}{l}\text { Self-efficacy for managing } \\
\text { chronic diseases (Six item } \\
\text { scale) }\end{array}$ & & $\mathbf{x}$ & $\mathbf{x}$ & $x$ \\
\hline $\begin{array}{l}\text { Medication adherence (Hill } \\
\text { Bone Compliance) }\end{array}$ & & $x$ & $\mathbf{x}$ & $\mathbf{x}$ \\
\hline \multicolumn{5}{|l|}{ Church leaders only } \\
\hline $\begin{array}{l}\text { Organisational readiness for } \\
\text { implementing change }\end{array}$ & $x$ & & & $x$ \\
\hline $\begin{array}{l}\text { Feasibility of Intervention } \\
\text { Measures }\end{array}$ & $x$ & & & $x$ \\
\hline $\begin{array}{l}\text { Acceptability of Intervention } \\
\text { Measure }\end{array}$ & $X$ & & & $x$ \\
\hline
\end{tabular}

will ensure that we assess further adaptations of various protocol elements. The potential for maintenance will be assessed using the Programme Sustainability Assessment Tool which will be delivered to divisional leaders via an online interview. Acceptability and feasibility of the intervention will be assessed using the following quantitative assessment tools: Feasibility of Intervention Measure, the Acceptability of Intervention Measure. Leaders of the FBOs or their designate will undergo a virtual interview where they will be asked questions based on these tools. Interviews will be audio recorded and transcribed verbatim.

\section{Data management, safety and confidentiality}

All quantitative data will be collected directly onto an encrypted tablet computer during the telephone interviews. At the end of each day, interview data will be uploaded (using a secure SSL connection) to a data server that is compliant with the Health Insurance Portability and Accountability Act (HIPAA), and data will be wiped from the data collection tablet. The data collection infrastructure will use the Research Electronic Data Capture (REDCap) tool - a secure web application for building and managing online surveys and databases. While REDCap can be used to collect virtually any type of data, it is specifically geared to support online and offline data capture for research studies and operations. Quantitative data will be deidentified before being cleared for analysis and analysed data will be aggregated at all times. Individual-level data access with identifiable information will be limited to the study investigators, research assistants and study manager. After study completion, deidentified aggregate data will be made available to participants on request and will be made publicly available if required during the peer-review publication process. The Zoom online platform will be used for delivery of the workshops. Sessions will not be recorded and do not form part of the data collection. To limit security and data protection problems, two factor authentication will be used and participants will be encouraged to enter the virtual room using their web browser. Data collected via WhatsApp will be anonymised and information will not be externalised or shared outside of the research group. Administrators will be required to monitor the chat and delete identifiable information shared in error. Data on WhatsApp are 
encrypted and saved chats will be de-identified, encrypted and saved on a secure cloud server.

\section{Analysis of primary and secondary outcomes}

Our primary outcome is mean SBP which will be measured at baseline and 6 months post-CDSMP workshops. We will determine the mean changes in SBP levels (primary endpoint) for the intervention group vs the control and compare differences in outcomes using mixed effects regression models. This model will allow for the effect of clustering on the precision of the estimates while allowing also for adjustment of covariates. Covariates will be analysed at both the cluster level: church size, denomination, previous existence of a health committee/programme and individual level. The latter will be guided by the socioecological framework using variables such as age, sex, educational level, marital status, income, health sector usage. We will adopt the intention-to-treat principle to analyse the data collected. Secondary outcomes will be assessed as follows: mean change in weight $(\mathrm{kg})$ will be compared between intervention and control groups after adjustment for covariates. Changes in dietary behaviour will be monitored by the calculation of the DASH diet score. Score ranges from 0 to 9 . A low score represents a poor diet for hypertension control. The higher the score the more appropriate for hypertension control. Physical activity will be measured by comparing the intervention group median physical activity in metabolic equaivalents (MET)-minutes per week. Using the results of the Global Physical Activity Questionnaire median physical activity in MET-minutes per week will be calculated before the intervention as well as 6 months postintervention. Perceived self-efficacy scores will be calculated Score ranges from 1 to 10. A higher score is better. Finally, medication adherence will be assessed before and after the intervention using the Hill Bone compliance scale. Both primary and secondary outcome measures will be taken preimplementation, immediately postworkshop and 6 months postworkshop. A two-sided $\mathrm{p}<0.05$ will be considered statistically significant. Baseline characteristics between the intervention and control groups will be described using proportions for categorical variables and means for continuous variables.

Additionally, WhatsApp groups will be created via which messages will be sent to participants encouraging use of blood pressure lowering medication and reinforcing messages learnt in the workshops. Workshop leaders will be responsible for posting weekly messages encouraging medication adherence and all participants will be allowed to post. Rules guiding confidentiality and security of posts will be provided at the start. At the end of the 6 -week period of the workshop and WhatsApp messaging the entire chat will be downloaded into a secure folder. The metadata for the chats will be analysed comparing group activity levels over time. Additionally, group chats will be separated by participant to examine metrics such as number of posts per participant, change in postfrequency over time as well as content and quality of posts. A previous study in Barbados showed that participants in a community-based project in churches preferred WhatsApp as a social-media based peer support system, indicating the feasibility of this approach. ${ }^{34}$ The qualitative thematic analysis of the WhatsApp group chats will be exploratory to determine potential role of this tool in behavioural change. We propose the use of the self-management theory framework to guide understanding of the themes emerging around barriers and facilitators of medication adherence. These methods have been informed by the Spirit Reporting Guidelines (online supplemental appendix $3)^{35}$ and are summarised in accordance with WHO Trial Registration Data Set (online supplemental appendix 4).

\section{Ethics and dissemination policy}

This project has received ethical approval from the University of the West Indies at Cave Hill/Ministry of Health and Wellness Institutional Review Board (IRB).A steering committee has been established to monitor study adherence to protocols and report adverse events (AEs) to IRB. The committee will operate independent of the funding source. The responsibility of collecting AE data, assessing, reporting and managing solicited and spontaneously reported AEs and other unintended effects of trial interventions or trial conduct will fall to an independent member of the steering committee. Given the study duration of 1 year and one postintervention data collection point, no interim analysis will be conducted.

\section{Potential AEs}

All persons whose blood pressure is taken, whether included in the study or not will be given a record to take to their primary care physician stating the findings. If blood pressure elevation is greater than 180 (SBP) or greater than 110 (diastolic blood pressure) clients will be immediately referred for urgent care to an emergency centre where an assessment of end organ damage can be conducted.

We plan to disseminate using national, regional and international conferences, peer-reviewed manuscripts, and the development of policy briefs to inform government policy around blood pressure control in community settings. We will provide FBO leaders of with guidelines on developing health programmes based on lessons learnt from this study. We will adhere to authorship guidelines of the International Committee of Medical Journal Editors and have no plan to use professional writers. All data will be kept confidentially on REDCap, a HIPAA and General Data Protection Regulation (GDPR) compliant platform operated by the University of the West Indies.

\section{Patient and public involvement}

The community-based nature of this study meant that the leaders of several churches have been involved in conceptualising the study before the protocol was submitted for funding. The leaders expressed a desire for the workshops being offered and identified assessment of physical activity and diet as priorities for the organisation. Their support was demonstrated in submission of a letter of support to the funders. The churches have provided 
volunteers (doctors, nurses and community aides) to be trained to assist with blood pressure screening and to be trained as workshop leaders for the intervention divisions. Initial pilot interviews with FBO leaders indicate that the self-management intervention is necessary and welcomed but they were unsure if persons would be willing to commit the necessary 2.5 hours per week to the programme. Health committees have been developed within churches whose remit will involve dissemination of the findings of this and other health programmes.

\section{Potential challenges and limitations}

There are some limitations and challenges that have the potential to impact study operations and specifics techniques to mitigate are required. The first is ensuring initial participation for screening and randomisation. We intend to use marketing strategies informed by experiences of key stakeholders to attract the participants who will engage in, and benefit from, the programmes. Workshop attendance will be encouraged by including consenting participants in WhatsApp groups which provide regular and systematic reminders of workshop dates. Attempts will be made to minimise loss to follow-up to less than $5 \%$ by ensuring multiple modes of contact are shared including work and home addresses. Participants will be contacted three times for each assessment before being considered lost to follow-up. The existence of the social network natural to the FBO community can also serve to minimise loss to follow-up.

The female predominance in churches limits this studies' potential to impact males. On recruitment days, female members will be specifically encouraged to bring their spouses and other adult males living in their household for screening. To reduce contamination treatment optimisation will be done to increase the chance that any change in SBP is due to the intervention and not to postintervention optimisation occurring at their usual medical care practice. Finally, we anticipate that COVID-19 protocols which may include repeated lockdowns and social distancing rules which minimise face-to-face participation will negatively impact recruitment increasing the risk of not reaching the required sample size.

This study leverages the social interactions of FBOs with the community to deliver an evidenced-based intervention that has been modified for cultural relevance and to emphasise medication adherence. The outcome is likely to be improved blood pressure management achieved by placing focus on the delivery of community-based components of the Chronic Care model. The lessons learnt from island-wide implementation in churches in Barbados have the potential to inform culturally similar settings in other SIDS.

\footnotetext{
Author affiliations

${ }^{1}$ George Alleyne Chronic Disease Research Centre, Caribbean Institute for Health

Research, The University of the West Indies, Bridgetown, Barbados

${ }^{2}$ Centre for Humanitarian Emergencies, Emory University School of Medicine, Atlanta, Georgia, USA

${ }^{3}$ Epidemiology Research Unit, Caribbean Institute for Health Research, The University of the West Indies, Kingston, Jamaica
}

Acknowledgements We thank the leaders and members of the faith-based organisations that have been involved in the design of this study and look forward to the contribution of the volunteer members who have committed to assisting with screening blood pressure measurements and delivery of workshops.

Contributors Conceived the study background, aims, setting: NS, IRH, SH, SGA, LB-G, KL and TSF; Contributed data analysis tools: NS, IRH, SH and TF; Designing the analysis: NS, IRH and SH; Drafting and finalising the manuscript: NS, IRH, SH, SGA, LB-G, KL and TF.

Funding This work is supported by Harvard T.H. Chan School of Public Health, Department of Global Health and Population, Bernard Lown Scholars in Cardiovascular Health Programme, grant number BLSCHP-2008 and the Appeal Fund of the George Alleyne Chronic Disease Research Centre, University of the West Indies which has not assigned a grant number. Dr. Saria Hassan is supported by a grant from the National Heart, Lung and Blood Institute 5K23HL152368. Lown Scholar Funder Contact info: Michele Hudak, Senior Grant Manager, +1 617-9986012 (p) mhudak@hsph.harvard.edu.

Disclaimer The funders will play no role in the collection, management, analysis, and interpretation of data; writing of the report; and the decision to submit the report for publication. They have no authority over any of these activities.

Competing interests None declared.

Patient consent for publication Not applicable.

Provenance and peer review Not commissioned; externally peer reviewed.

Supplemental material This content has been supplied by the author(s). It has not been vetted by BMJ Publishing Group Limited (BMJ) and may not have been peer-reviewed. Any opinions or recommendations discussed are solely those of the author(s) and are not endorsed by BMJ. BMJ disclaims all liability and responsibility arising from any reliance placed on the content. Where the content includes any translated material, BMJ does not warrant the accuracy and reliability of the translations (including but not limited to local regulations, clinical guidelines, terminology, drug names and drug dosages), and is not responsible for any error and/or omissions arising from translation and adaptation or otherwise.

Open access This is an open access article distributed in accordance with the Creative Commons Attribution Non Commercial (CC BY-NC 4.0) license, which permits others to distribute, remix, adapt, build upon this work non-commercially, and license their derivative works on different terms, provided the original work is properly cited, appropriate credit is given, any changes made indicated, and the use is non-commercial. See: http://creativecommons.org/licenses/by-nc/4.0/.

\section{ORCID iDs}

Natasha P Sobers http://orcid.org/0000-0002-9444-0706

lan Richard Hambleton http://orcid.org/0000-0002-5638-9794

Saria Hassan http://orcid.org/0000-0003-0700-9529

Simon George Anderson http://orcid.org/0000-0002-8896-073X

Trevor S Ferguson http://orcid.org/0000-0002-2393-1452

\section{REFERENCES}

1 GBD 2015 Risk Factors Collaborators. Global, regional, and national comparative risk assessment of 79 behavioural, environmental and occupational, and metabolic risks or clusters of risks, 1990-2015: a systematic analysis for the global burden of disease study 2015. Lancet 2016;388:1659-724.

2 Sarki AM, Nduka CU, Stranges S, et al. Prevalence of hypertension in low- and middle-income countries: a systematic review and metaanalysis. Medicine 2015;94:e1959.

3 Howitt C, Hambleton IR, Rose AMC, et al. Social distribution of diabetes, hypertension and related risk factors in Barbados: a crosssectional study. BMJ Open 2015;5:e008869.

4 Institute for Health Metrics and Evaluation. Global health data exchange: GBD results tool, 2019. Available: http://ghdx.healthdata. org/gbd-results-tool

5 Bertram MY, Sweeny K, Lauer JA, et al. Investing in noncommunicable diseases: an estimation of the return on investment for prevention and treatment services. Lancet 2018;391:2071-8.

6 Foster N, Atkins V, Hutton M, et al. Ncds and trade policy in the Caribbean: informing civil society advocacy for action. Bridgetown, Barbados, 2017.

7 Chodosh J, Morton SC, Mojica W, et al. Meta-analysis: chronic disease self-management programs for older adults. Ann Intern Med 2005;143:427-38. 
8 Vas A, Devi ES, Vidyasagar S, et al. Effectiveness of selfmanagement programmes in diabetes management: a systematic review. Int J Nurs Pract 2017;23. doi:10.1111/ijn.12571. [Epub ahead of print: 31072017$]$.

9 Werfalli M, Raubenheimer P, Engel M, et al. Effectiveness of community-based peer-led diabetes self-management programmes (COMP-DSMP) for improving clinical outcomes and quality of life of adults with diabetes in primary care settings in low and middleincome countries (LMIC): a systematic review and meta-analysis. BMJ Open 2015;5:e007635.

10 Brady TJ, Murphy L, O'Colmain BJ, et al. A meta-analysis of health status, health behaviors, and health care utilization outcomes of the chronic disease self-management program. Prev Chronic Dis 2013;10:120112.

11 Lorig KR, Ritter P, Stewart AL, et al. Chronic disease selfmanagement program: 2-year health status and health care utilization outcomes. Med Care 2001;39:1217-23.

12 Dodani S, Sullivan D, Pankey S, et al. Heals: a faith-based hypertension control and prevention program for African American churches: training of church leaders as program Interventionists. Int $J$ Hypertens 2011;2011:1-7.

13 Schoenthaler AM, Lancaster KJ, Chaplin W, et al. Cluster randomized clinical trial of faith (faith-based approaches in the treatment of hypertension) in blacks. Circ Cardiovasc Qual Outcomes 2018;11:e004691.

14 Go B. Population and housing census. In: Barbados statistical service. 2013. Bridgetown: Government of Barbados, 2010.

15 Gwadry-Sridhar FH, Manias E, Lal L, et al. Impact of interventions on medication adherence and blood pressure control in patients with essential hypertension: a systematic review by the ISPOR medication adherence and persistence special interest group. Value Health 2013:16:863-71.

16 Ebrahim S, Detection ES. Detection, adherence and control of hypertension for the prevention of stroke: a systematic review. Health Technol Assess 1998;2:1-78.

17 Government of Barbados. About barbados: demographics bridgetown, Barbados: barbados integrated government, 2019. Available: https://www.gov.bb/Visit-Barbados/demographics [Accessed 14 Sep 2019].

18 Whelton PK, Carey RM, Aronow WS, et al. 2017 ACC/AHA/AAPA ABC/ACPM/AGS/APhA/ASH/ASPC/NMA/PCNA guideline for the prevention, detection, evaluation, and management of high blood pressure in adults: a report of the American College of Cardiology/ American heart association Task force on clinical practice guidelines. J Am Coll Cardiol 2018;71:e127-248.

19 Pan American Health Organization. Virtual course on accurate automated blood pressure measurement (2020), 2020. Available: https://www.campusvirtualsp.org/en/course/virtual-course-accurateautomated-blood-pressure-measurement-2020
20 Barbados Government. Official Gazette: emergency management (COVID-19) churches (no. 3) directive. Barbados: Barbados Government Printing Department, 2020.

$21 \mathrm{He} \mathrm{J}$, Irazola V, Mills KT, et al. Effect of a community health WorkerLed multicomponent intervention on blood pressure control in low-income patients in Argentina: a randomized clinical trial. JAMA 2017;318:1016-25.

22 Appel LJ, Champagne CM, Harsha DW, et al. Effects of comprehensive lifestyle modification on blood pressure control: main results of the premier clinical trial. JAMA 2003;289:2083-93.

23 Campbell MK, Mollison J, Steen N, et al. Analysis of cluster randomized trials in primary care: a practical approach. Fam Pract 2000;17:192-6.

24 StataCorp. Stata statistical software: release 15. College Station, TX: StataCorp LLC, 2017.

25 Self-Management Resource Center. Chronic disease selfmanagement (CDSMP), 2021. Available: https://www.selfmana gementresource.com/programs/small-group/chronic-disease-selfmanagement

26 Lorig K, Holman HR, Sobel DS. Living a healthy life with chronic conditions. In: Boulder. 4 edn. Colorado: Bull Publishing Company, 2012: 343

27 Wiltsey Stirman S, Baumann AA, Miller CJ. The frame: an expanded framework for reporting adaptations and modifications to evidencebased interventions. Implement Sci 2019;14:58.

28 Bank W. Mobile cellular subscriptions (per 100 people) - Barbados 2019.

29 National Cancer Institute. Division of cancer control and population sciences. dietary screener questionnaires (DSQ) in the nhanes 2009-10: DSQ 2020. Available: https://epi.grants.cancer.gov/nhanes/ dietscreen/questionnaires.html

30 Fung TT, Chiuve SE, McCullough ML, et al. Adherence to a dashstyle diet and risk of coronary heart disease and stroke in women. Arch Intern Med 2008;168:713-20.

31 Lorig KR, Sobel DS, Ritter PL, et al. Effect of a self-management program on patients with chronic disease. Eff Clin Pract 2001:4:256-62.

32 Kim MT, Hill MN, Bone LR, et al. Development and testing of the Hill-Bone compliance to high blood pressure therapy scale. Prog Cardiovasc Nurs 2000;15:90-6.

33 Glasgow RE, Vogt TM, Boles SM. Evaluating the public health impact of health promotion interventions: the RE-AIM framework. Am J Public Health 1999;89:1322-7.

34 Lambton-Howard D, Simpson E, Quimby K. Blending into everyday life: designing a social media-based peer support system. $\mathrm{CHI}$ Conference on Human Factors in Computing Systems (CHI '21), Yokohama, Japan, 2021:2021.

35 Chan A-W, Tetzlaff JM, Gøtzsche PC, et al. Spirit 2013 explanation and elaboration: guidance for protocols of clinical trials. $B M J$ 2013;346:e7586. 